Pacific Journal of Mathematics

EXTENDING LEBESGUE MEASURE BY INFINITELY MANY
SETS 


\title{
EXTENDING LEBESGUE MEASURE BY INFINITELY MANY SETS
}

\author{
TIM CARLSON
}

Consider the following question: under what conditions on a collection of subsets of the unit interval can the existence of an extension of Lebesgue measure defined on each element of the collection be guaranteed? The main purpose of this paper is to find conditions on the cardinality of the collection whose sufficiency can be shown consistent without the use of large cardinals. For example, if ZFC is consistent so is $\mathrm{ZFC}+$ "Lebesgue measure can be extended to any countable collection of sets".

The results of this paper complement work of earlier researchers. Banach and Kuratowski showed that assuming the continuum hypothesis there is a countable collection of sets of reals for which no extension exists. Solovay proved that an extension of Lebesgue measure to all sets is equiconsistent with the existence of a measurable cardinal.

1. Definitions and notation. Almost all definitions and notations are standard but I will make a few remarks in hopes of avoiding any problems.

${ }^{X} Y$ denotes the collection of functions from $X$ into $Y$. The concatenation of two sequences $s$ and $t$ is written as $s^{\wedge} t .\left\langle a_{l}: i \in I\right\rangle$ denotes the function $i \mapsto a_{i}$ with domain I. CBA is short for complete Boolean algebra. If $T$ is a tree then subtrees are always intended to be closed downward (trees grow upward). A tree $T$ is $<\rho$ branching for a cardinal $\rho$ if the collection of immediate successors of an element in $T$ always has cardinality less than $\rho$. If $\mathscr{C}$ is a complete subalgebra of a CBA $\mathscr{B}$ and $G$ is a $V$-generic ultrafilter on $\mathscr{C}$, I use $\mathscr{B} / G$ for the quotient of $\mathscr{B}$ by the ideal generated in $\mathscr{B}$ by the dual of $G$.

Jech [4] is a good reference for the set theoretic aspects of this paper.

Given a set $X$ and a cardinal $\rho$, a $\rho$-algebra on $X$ is an algebra of subsets of $X$ which is closed under unions of size less than $\rho$. $\sigma$-algebra means the same as $\omega_{1}$-algebra as usual. Measures are only assumed to be finitely additive and with a domain consisting of an algebra of sets (not necessarily a $\sigma$-algebra). Suppose $\mathscr{A}$ is an algebra of subsets of $X$ and $\nu$ is a measure defined on $\mathscr{A}$. I will always tacitly assume that the measure of each element in $\mathscr{A}$ is in $[0,1]$ and $\nu(X)=1 . \nu$ is $\rho$-additive if whenever $A_{l} \in \mathscr{A}$ for $i \in I$ are pairwise disjoint with $\bigcup_{l \in I} A_{l}=X$ and $|I|<\rho$ then $\sum_{l \in I} \nu\left(A_{l}\right)=1$.

Halmos [3] may be used for the basic facts on product measures. 
2. Codes and measure theoretic preliminaries. We will often want to discuss the $\rho$-algebra generated by a collection of sets. For this the notion of code is useful. Fix a list $v_{k}(k \in \mathrm{On})$ of variables.

Definition 1. Assume $\rho$ is an infinite cardinal. A $\rho$-code is a pair $(T, e)$ where $T$ is a well-founded subtree of $\rho^{<\omega}$ which is $<\rho$ branching and $e$ is a function which assigns variables to the maximal nodes of $T$. $(T, e)$ is a code if $(T, e)$ is a $\rho$-code for some $\rho$. The rank of $(T, e)$ is defined to be the rank of $T$ in the usual sense.

Note that if $\rho$ is regular then $|T|<\rho$ and $(T, e)$ has fewer than $\rho$ variables.

Definition 2. Suppose $c=(T, e)$ is a code. If $s \in T$ define $c_{s}=(\{t$ : $\left.\left.s^{\wedge} t \in T\right\}, f\right)$ where $f(t)=e\left(s^{\imath} t\right)$. If $A_{k} \subseteq X$ for $k \in \alpha$ and all the variables of $c$ are among $v_{k}(k \in \alpha)$ then $c\left(\left\langle A_{k}: k \in \alpha\right\rangle\right)$ is defined by induction of the rank of $c$. If the rank of $c$ is 0 then \langle\rangle is the only element of $T$ and $e(\langle\rangle)=v_{i}$ for some $i \in \alpha$. In this case define $c\left(\left\langle A_{k}: k \in \alpha\right\rangle\right)=$ $A_{l}$. If the rank of $C$ is not 0 define $c\left(\left\langle A_{k}: k \in \alpha\right\rangle\right)$ to be

$$
\bigcup\left\{X-c_{(\langle i\rangle)}\left(\left\langle A_{k}: k \in \alpha\right\rangle\right):\langle i\rangle \in T\right\} .
$$

Similarly, if $\mathscr{B}$ is a CBA and $b_{k} \in \mathscr{B}$ for $k \in \alpha$ define $c\left(\left\langle b_{k}: k \in \alpha\right\rangle\right)$.

Fact 1 . Suppose $\mathscr{A}$ is a nonempty collection of subsets of a fixed set $X$ and $\rho$ is a regular cardinal. The $\rho$-algebra on $X$ generated by $\mathscr{A}$ consists exactly of the sets obtained by evaluating $\rho$-codes at sequences from $\mathscr{A}$.

Fact 2. If $c$ is a code, $\mathscr{B}$ is a CBA, $\underline{x}, \underline{A} \in V^{\mathscr{B}}, \underline{A}_{k} \in V^{\mathscr{B}}$ for $k \in \alpha$ and $V^{\mathscr{B}} \vDash " \underline{A}_{k} \subseteq \underline{A}$ " then

$$
\left\|\underline{x} \in c\left(\left\langle\underline{A}_{k}: k \in \alpha\right\rangle\right)\right\|=c\left(\left\langle\left\|\underline{x} \in \underline{A}_{k}\right\|: k \in \alpha\right\rangle\right) .
$$

All the forcing done in this paper uses measure algebras. The basic facts needed are discussed next. The reader is assumed to have some familiarity with the usual product measure on ${ }^{x_{2}}$ where both 0 and 1 are given probability $1 / 2$. This measure is defined on the $\sigma$-algebra of Baire sets which is the $\sigma$-algebra generated by the basic open sets in the product topology where $\{0,1\}$ is given the discrete topology.

Definition 3. Fix a set $X$. Let $\mu_{X}$ be the usual product measure on ${ }^{X_{2}}$ and define $\mathscr{A}(X)$ to be the collection of Baire sets, $\mathscr{B}(X)$ is the corresponding measure algebra, i.e. $\mathscr{B}(X)$ is the quotient of $\mathscr{A}(X)$ by the ideal 
of measure zero sets. For $x \in X$ define the canonical generator at $x$ to be the element of $\mathscr{B}(X)$ represented by $\left\{f \in{ }^{X} 2: f(x)=1\right\}$.

Each canonical generator has measure $1 / 2$. The collection of canonical generators is independent and countably generates $\mathscr{B}(X)$.

Definition 4. Assume $s \subseteq Y$. A set $B \in \mathscr{A}(X)$ has support $s$ provided whether $f$ is in $A$ depends only on $f \uparrow s . b \in \mathscr{B}(X)$ has support $s$ if some element in $b$ does.

Clearly, all Baire sets have countable support implying that all elements of $\mathscr{B}(X)$ have countable support.

Notice that if $Y \subseteq X$ there's a canonical complete embedding of $\mathscr{B}(Y)$ into $\mathscr{B}(X)$. This embedding also preserves measure. Henceforth, I identify $\mathscr{B}(Y)$ with a complete subalgebra of $\mathscr{B}(X)$ in this way. Moreover, I will write $\mu$ rather than $\mu_{X}$ since no confusion is likely and will reserve $\mu$ only for product measures.

Fact 3. Assume $X \subseteq Y$ and $G \subseteq \mathscr{B}(X)$ is $V$-generic. $\mathscr{B}(Y) / G$ is a measure algebra in $V[G]$ as witnessed by $\nu$ where

$$
\nu(b / G)=\lim _{c \in G}(\mu(b \cdot c) / \mu(c)) .
$$

In fact, if $b_{y}$ is the canonical generator at $y$ in $\mathscr{B}(Y)$ for $y \in Y$ then $\nu\left(b_{y} / G\right)=1 / 2$ for $y \in Y-X$ and the collection $b_{y} / G(y \in Y-X)$ is independent and countably generates $\mathscr{B}(Y) / G$ in $V[G]$.

Fact 3 shows that $\mathscr{B}(Y) / G$ is isomorphic to $\mathscr{B}(Y-X)^{V[G]}$ by a unique isomorphism which sends $b_{y} / G$ to the canonical generator at $y$ in $\mathscr{B}(Y-X)^{V[G]}$ for $y \in Y-X$.

I will need a generalization of Fact 2.

Fact 4. Assume $X \subseteq Y$ and $G \subseteq \mathscr{B}(X)$ is $V$-generic. If $\underline{c} \in V^{\mathscr{B}(X)}$ is a name for a code and $\underline{A}_{k} \in V^{\mathscr{B}(Y)}$ is a name for a subset of $\underline{A} \in V^{\mathscr{B}(Y)}$ for $k \in \alpha$ then for any name $\underline{x}$

$$
\left\|\underline{x} \in \underline{c}\left(\left\langle\underline{A}_{k}: k \in \alpha\right\rangle\right)\right\| / G=c\left(\left\langle\left\|\underline{x} \in \underline{A}_{k}\right\| / G: k \in \alpha\right\rangle\right)
$$

where $c$ is the interpretation of $\underline{c}$.

Fact 4 is proved in $V[G]$ by induction on the rank of $c$.

Finally, I will need

Fact 5. Assume $X \subseteq Y \subseteq Z$ and $G \subseteq \mathscr{B}(Y)$ is $V$-generic. Set $H=$ $G \cap \mathscr{B}(X)$. If $b \in \mathscr{B}(X \cup(Z-Y))$ then $\mu(b / G)=\mu(b / H)$. 
Sketch the proof. Note that this is trivial if $X$ is empty since

$$
\mu(b / G)=\lim _{c \in G} \frac{\mu(b \cdot c)}{\mu(c)}=\lim _{c \in G} \frac{\mu(b) \cdot \mu(c)}{\mu(c)}=\mu(b)
$$

$(\mu(b \cdot c)=\mu(b) \cdot \mu(c)$ since $c$ and $b$ have disjoint supports). Although a proof for $X \neq \varnothing$ can be given along these lines and this is the basic intuition, a modification is more economical. Namely, working in $V[G]$ show that the collection of $b$ for which the theorem holds contains all finite Boolean combinations of the canonical generator of $\mathscr{B}(X \cup(Z-Y))$ and is closed under complements and increasing unions of sequences in $V$.

Suppose $G \subseteq \mathscr{B}(\theta)$ is $V$-generic and define $x: \theta \rightarrow 2$ by $x(i)=1$ iff the canonical generator at $i$ is in $G$. In this case $V[G]=V[x]$. If $\theta=\omega$ then $x$ is called a random real over $V . \mathscr{B}(\omega)$ is often referred to as the CBA for adding a random real and $\mathscr{B}(\theta)$ as the CBA for adding $\theta$ random reals if $\theta>\omega$.

The main theorem of this paper is in $\$ 4$. It involves defining a measure in $V^{\mathscr{B}(\theta)}$ for some $\theta$. Often the measure will be essentially on a cardinal $\kappa$ below $\theta$, but to illustrate a point suppose the measure to be defined is on $\theta$. The problem is how to choose the measure of some subset $\underline{A}$ of $\theta$ in $V^{\mathscr{B}(\theta)}$. Consider the canonical name $\underline{x}$ for the generic subset of $\theta$ described above. $\|\alpha \in \underline{x}\|(\alpha \in \theta)$ all have measure $1 / 2$ and they are independent. A reasonable attempt would be to assign measure $1 / 2$ to $\underline{x}$. This is precisely the main idea for defining the measure in $V^{\mathscr{B}(\theta)}$ : choose a "large" subset $E$ of $\theta$ and force with a small subalgebra to get the reduced values of $\|\alpha \in \underline{A}\|(\alpha \in E)$ to be independent and so that they all have the same measure. This method is equivalent to the usual technique (see [4], pp. 423-425 for example). Although the modifications made here lengthen the proofs slightly, I think they result in a better understanding of the resulting measure.

3. A lemma concerning normal filters. The following lemma will be used to generate measures in the next section.

LeMMa. Assume $\kappa$ is a regular uncountable cardinal, $D$ is a normal filter on $\kappa, \lambda$ is a cardinal below $\kappa, \rho$ is regular cardinal below $\kappa, \chi^{<\rho}<\kappa$ for each cardinal $\chi<\kappa$ and $S$ is positive $\bmod D$ where $S \subseteq\{\alpha \in \kappa: \operatorname{cof}(\alpha) \geq \rho\}$. If $f_{\xi}: S \rightarrow \kappa$ is regressive for $\xi \in \lambda$ then there's a nontrivial $\rho$-complete filter $D^{*}$ extending $D$ such that each $f_{\xi}$ is constant on an element of $D^{*}$. 
Proof. For each $\alpha \in S$ define $h_{\alpha}: \lambda \rightarrow \alpha$ by $h_{\alpha}(\xi)=f_{\xi}(\alpha)$ and let $D_{\alpha}$ be the filter generated by $D$ along with the sets $\left\{\beta \in S: h_{\beta} \uparrow A=h_{\alpha} \uparrow A\right\}$ where $A$ ranges over $[\lambda]^{<\rho}$. Evidently, $D_{\alpha}$ is $\rho$-complete. Moreover, if $\xi \in \lambda$ then $f_{\xi}$ is constant on $\left\{\beta \in S: h_{\beta}(\xi)=h_{\alpha}(\xi)\right\}$ which is in $D_{\alpha}$. Therefore, if $D_{\alpha}$ is non-trivial for some $\alpha$ the lemma is proved.

Assume $D_{\alpha}$ is trivial for all $\alpha \in S$. This means that for $\alpha \in S$ there exists $A_{\alpha} \in[\lambda]^{<\rho}$ such that $\left\{\beta \in S: h_{\beta} \uparrow A_{\alpha}=h_{\alpha} \uparrow A_{\alpha}\right\}$ is measure zero $\bmod D$. Define a regressive function $F: S \rightarrow \kappa$ by

$$
F(\alpha)=\sup \left\{h_{\alpha}(\xi)+1: \xi \in A_{\alpha}\right\} .
$$

By the normality of $D$, choose $S_{0} \subseteq S$ which is positive such that $F$ is constant on $S_{0}$. Let $\chi$ be the constant value of $F$ on $S_{0}$. If $\alpha \in S_{0}$ then $h_{\alpha} \uparrow A_{\alpha}$ is a subset of $\lambda \times \chi$ of size $<\rho$. There are fewer than $\kappa$ such functions. Since $D$ is $\kappa$-complete, there's a positive subset $S_{1}$ of $S_{0}$ such that $h_{\alpha} \uparrow A_{\alpha}=h_{\beta} \uparrow A_{\beta}$ for $\alpha, \beta \in S_{1}$. This contradicts the choice of $A_{\alpha}$ for each $\alpha \in S_{1}$.

For the purpose of this paper only the case $\rho=\omega$ and $D$ is the closed unbounded filter on $\kappa$ is necessary.

4. The consistency of measure extension principles. For the sake of readability, I will simplify cardinal arithmetic in this section by assuming GCH. I invite the reader to draw diagrams of the systems of supports in $\kappa \times \theta$ as they arise in the proof.

LEMma. Assume GCH. If $\theta$ is an infinite cardinal then $V^{\mathscr{B}(\theta)} \vDash$ "if $\omega<\rho=\operatorname{cof}(\lambda)$ and $\lambda<\theta$ then whenever $\mathscr{C}$ is a collection of subsets of ${ }^{\theta} 2$ of size $\lambda$ there is a $\rho$-additive extension of the usual product measure to a $\rho$-algebra containing $\mathscr{C} "$.

Proof. Assume GCH and that $\theta$ is an infinite cardinal. Suppose also that $\omega<\rho=\operatorname{cof}(\lambda)$ and $\lambda$ is a cardinal below $\theta$. Set $\kappa=\lambda^{+}$. Since $\mathscr{B}(\kappa \times \theta)$ is isomorphic to $\mathscr{B}(\theta)$, it suffices to show $V^{\mathscr{B}} \vDash$ "if $\mathscr{C}$ is a collection of subsets of ${ }^{\theta} 2$ of size $\lambda$ there is a $\rho$-additive extension of the usual product measure to a $\rho$-algebra containing $\mathscr{C}$ " where $\mathscr{B}=\mathscr{B}(\kappa \times \theta)$.

Assume $\underline{\mathscr{C}} \in V^{\mathscr{B}}$ and $V^{\mathscr{B}} \vDash " \underline{\mathscr{C}}$ is a collection of subsets of ${ }^{\theta} 2$ of size $\lambda$ ”. Choose $\underline{C}_{\eta} \in V$ for $\eta \in \lambda$ such that $V^{\mathscr{B}} \vDash$ “ $\underline{\mathscr{C}}=\left\{\underline{C}_{\eta}: \eta \in \lambda\right\}$ ”.

The first step to extending the measure in $V^{\mathscr{B}}$ is to cut down to a set of size $\kappa$ which has outer measure 1. For $\alpha \in \kappa$ define $\underline{x}_{\alpha} \in V^{\mathscr{B}}$ such that $V^{\mathscr{B}} \vDash$ " $\underline{x}_{\alpha}: \theta \rightarrow 2$ " by taking the $\alpha$ column of the generic set, i.e. $V^{\mathscr{B}}$ $\vDash$ " $\underline{x}_{\alpha}(i)=1$ iff the canonical generator corresponding to $(\alpha, i)$ is in $\underline{G}$ ". 
For $\alpha \in \kappa$ and $\eta \in \lambda$ define $b_{\alpha \eta} \in \mathscr{B}$ to be $\left\|\underline{x}_{\alpha} \in \underline{C}_{\eta}\right\|$ and choose a countable support $s_{\alpha \eta}$ for $b_{\alpha \eta}$. We may assume that all the $s_{\alpha \eta}$ are subsets of $\kappa \times \kappa$ since the ordering of $\theta$ is irrelevant to the hypothesis. Set $S=\{\alpha \in \kappa: \operatorname{cof}(\alpha)=\rho, \lambda<\alpha$ and $\alpha$ is closed under the Godel pairing function $\}$ and let $D$ be the club filter on $\kappa$. Let $B_{\xi}(\xi \in \lambda)$ enumerate $[\lambda]^{<\rho}$.

I now want to define "regressive" functions $f_{\xi}$ on $S$ for $\xi \in \lambda$ so that $f_{\xi}(\alpha)$ codes the structure of the collection of $b_{\alpha \eta}\left(\eta \in B_{\xi}\right)$ along with $\left\|\underline{x}_{\alpha}(i)=1\right\|(i \in \alpha)$. Set $s_{\alpha \xi}^{*}=\bigcup\left\{s_{\alpha \eta}: \eta \in B_{\xi}\right\}$. Choose $\beta$ minimal such that $s_{\alpha \xi}^{*} \cap((\alpha+1) \times \alpha)$ is contained in $(\beta \times \beta) \cup(\{\alpha\} \times \beta)$. Now collapse $s_{\alpha \xi}^{*}$ as follows. Let $\left.\tau=\mid s_{\alpha \xi}^{*}-(\alpha+1) \times \alpha\right) \mid$ and choose a bijection $h$ : $((\beta+1) \times \beta) \cup \tau \rightarrow(\beta \times \beta) \cup(\{\alpha\} \times \beta) \cup s_{\alpha \xi}^{*}$ such that $h$ is the identity on $\beta \times \beta$ and $h((\beta, i))=(\alpha, i)$. $h$ induces a complete embedding of $\mathscr{B}((\beta+1) \times \beta) \cup \tau$ into $\mathscr{B}$. For $\eta \in B_{\xi}$ let $b_{\alpha \eta}^{\prime}$ be the preimage of $b_{\alpha \eta}$ under this embedding. Define $f_{\xi}(\alpha)=\left(\beta, \tau,\left\langle b_{\alpha \eta}^{\prime}: \eta \in B_{\xi}\right\rangle\right) . f_{\xi}(\alpha)$ can be coded by an ordinal below $\alpha$ so that the lemma of the previous section applies. So let $D^{*}$ be a $\rho$-complete filter extending $D$ such that each $f_{\xi}$ is constant on an element of $D$.

Now to define the measure $\nu$. Assume $G \subseteq \mathscr{B}$ is $V$-generic. Since $\mathscr{B}$ is ccc, $D^{*}$ generates a $\rho$-complete filter in $V[G]$ which I also call $D^{*}$.

Work in $V[G]$ from now on.

The letters $X$ and $Y$ will always denote subsets of $\kappa \times \theta$ in $V$ which have cardinality at most $\lambda$. Define $G(X)$ to be $G \cap \mathscr{B}(X)$. If $\underline{A}$ is a name for a subset of ${ }^{\theta} 2$ define $\nu_{X}(\underline{A})$ to be

$$
\lim _{E \in D^{*}} \inf \left\{\mu\left(\left\|\underline{x}_{\alpha} \in \underline{A}\right\| / G(X)\right): \alpha \in E\right\} .
$$

Let $\mathscr{A}$ be the $\rho$-algebra generated by the collection of $C_{\eta}(\eta \in \lambda)$ along with the Baire sets where $C_{\eta}$ is the interpretation of the name $\underline{C}_{\eta}$. If $A \in \mathscr{A}$ choose a name $\underline{A}$ for $A$ and define $\nu(A)=\lim _{X} \nu_{X}(\underline{A})$. Clearly, if $\nu(A)$ is defined then it is independent of the choice of $\underline{A}$.

Claim 1. If $A \in \mathscr{A}$ is a name for $A$ then there exist $X$, a real number $r$ and $E \in D^{*}$ such that for all $Y$ containing $X, \mu\left(\left\|\underline{x}_{\alpha} \in \underline{A}\right\| / G(Y)\right)=r$ on a tail of $\alpha \in E$.

Assume $A \in \mathscr{A}$ and $\underline{A}$ is a name for $A$. For $i \in \theta$ define $N_{i}=\{x$ : $x(i)=1\}$. The $\rho$-algebra $\mathscr{A}$ is generated from $C_{\eta}(\eta \in \lambda)$ along with $N_{t}$ $(i \in \theta)$. However, $A$ is generated by fewer than $\rho$ elements among these. There exist $\xi \in \lambda$ and $B \subseteq \theta$ in $V$ of size less than $\rho$ such that $A$ is in the $\rho$-algebra generated by $C_{\eta}\left(\eta \in B_{\xi}\right)$ and $N_{i}(i \in B)$. Let $h: \rho \rightarrow B_{\xi} \cup B$ be in $V$ such that the even ordinals map onto $B_{\xi}$ and the odd ordinals map 
onto $B$. Now define a sequence $a$ with domain $\rho$ by $a(k)=C_{h(k)}$ if $k$ is even and $a(k)=N_{h(k)}$ if $k$ is odd and let $\underline{a}$ be the name

$$
\left\langle\underline{C}_{h(k)}: k \in \rho \text { and } k \text { even }\right\rangle \cup\left\langle\underline{N}_{h(k)}: k \in \rho \text { and } k \text { odd }\right\rangle
$$

for $a$ where $\underline{N}_{i}$ is the canonical name for $N_{l}$. Choose a $\rho$-code $c$ such that $c(a)=A$.

There is a set $E \in D^{*}$ on which $f_{\xi}$ is constant with value $\left(\beta, \tau,\left\langle d_{\eta}: \eta \in B_{\xi}\right\rangle\right)$ say. Choose $X$ such that $c$ has a name $\underline{c}$ in $V^{\mathscr{B}(X)}$, there's a condition in $G(X)$ forcing " $\underline{A}=\underline{c}(\underline{a})$ " and $\beta \times \beta \subseteq X$.

Suppose $Y$ contains $X$. It will suffice to show that $\mu\left(\left\|\underline{x}_{\alpha} \in \underline{A}\right\| / G(Y)\right)$ $=\mu\left(\left\|\underline{x}_{\alpha^{\prime}} \in \underline{A}\right\| / G(X)\right)$ for a tail of $\alpha$ and $\alpha^{\prime}$ in $E$. Choose $\delta$ large enough so that $Y \subseteq \delta \times \theta, Y \cap(\kappa \times \kappa) \subseteq \delta \times \delta$ and $B \cap \kappa \subseteq \delta$. Assume $\alpha, \alpha^{\prime} \in$ $E$ and $\delta \leq \alpha, \alpha^{\prime}$.

$$
\begin{aligned}
\left\|\underline{x}_{\alpha} \in \underline{A}\right\| / G(X) & =\left\|\underline{x}_{\alpha} \in \underline{c}(\underline{a})\right\| / G(X) \\
& =c\left(\left\langle\left\|\underline{x}_{\alpha} \in \underline{a}(k)\right\| / G(X): k \in \rho\right\rangle\right)
\end{aligned}
$$

by Fact 4 of $\S 2$. $\left\|\underline{x}_{\alpha} \in \underline{a}(k)\right\|$ has one of two forms: $\left\|\underline{x}_{\alpha} \in \underline{C}_{\eta}\right\|$ for some $\eta \in B_{\xi}$ or $\left\|\underline{x}_{\alpha} \in \underline{N}_{l}\right\|$ for some $i \in B$. In either case, $\left\|\underline{x}_{\alpha} \in \underline{a}(k)\right\|$ is in $\mathscr{B}(Z)$ where $Z=X \cup((\kappa \times \theta)-Y)$. So each $\left\|\underline{x}_{\alpha} \in \underline{a}(k)\right\| / G(X)$ is in $\mathscr{B}(Z) / G(X)$ implying $\left\|\underline{x}_{\alpha} \in \underline{A}\right\| / G(X)$ is also. By Fact 5 of $\S 2$

$$
\mu\left(\left\|\underline{x}_{\alpha} \in \underline{A}\right\| / G(X)\right)=\mu\left(\left\|\underline{x}_{\alpha} \in \underline{A}\right\| / G(Y)\right) .
$$

On the other hand, by the definition of $f_{\xi}$ and the choices of $E$ and $\delta$ there is an automorphism $\psi$ of $\mathscr{B}$ induced by a permutation $\kappa \times \theta$ which fixes $\mathscr{B}(X)$ and interchanges $\left\|\underline{x}_{\alpha} \in \underline{C}_{\eta}\right\|\left(=b_{\alpha \eta}\right)$ with $\left\|\underline{x}_{\alpha^{\prime}} \in \underline{C}_{\eta}\right\|\left(=b_{\alpha^{\prime} \eta}\right)$ for $\eta \in B_{\xi}$ and $\left\|\underline{x}_{\alpha} \in \underline{N}_{i}\right\|\left(=\left\|\underline{x}_{\alpha}(i)=1\right\|\right)$ with $\left\|\underline{x}_{\alpha^{\prime}} \in \underline{N}_{i}\right\|\left(=\left\|\underline{x}_{\alpha^{\prime}}(i)=1\right\|\right)$ for $i \in B$. This automorphism of $\mathscr{B}$ is measure preserving since it is induced by a permutation. $\psi$ induces a measure preserving automorphism $\psi^{*}$ of $\mathscr{B} / G(X)$ given by $\psi^{*}(b / G(X))=\psi(b) / G(X)$. Since

$$
\left\|\underline{x}_{\alpha} \in \underline{A}\right\| / G(X)=c\left(\left\langle\left\|\underline{x}_{\alpha} \in \underline{a}(k)\right\| / G(X): k \in \rho\right\rangle\right)
$$

and similar for $\alpha^{\prime}$ by Fact 4 of $\S 2$, it follows that $\psi^{*}$ interchanges $\left\|\underline{x}_{\alpha} \in \underline{A}\right\| / G(X)$ and $\left\|\underline{x}_{\alpha^{\prime}} \in \underline{A}\right\| / G(X)$ verifying that they have the same measure. Combining this with the above,

$$
\mu\left(\left\|\underline{x}_{\alpha} \in \underline{A}\right\| / G(Y)\right)=\mu\left(\left\|\underline{x}_{\alpha^{\prime}} \in \underline{A}\right\| / G(X)\right) .
$$

This finishes the proof of the claim.

Claim 2. $\nu$ is defined on every element of $A$.

This follows immediately from Claim 1. 
Claim 3. $\nu$ is $\rho$-additive.

Suppose $A_{j} \in \mathscr{A}(j \in \gamma)$ are pairwise disjoint and $\gamma<\rho$. Choose a sequence of names $\underline{A}_{j}(j \in \gamma)$ which are forced outright to be disjoint and choose a name $\underline{A}$ for the union. Chose $X_{i}, E_{j}$ and $r_{j}$ for $A$, and $\underline{A}$, by Claim 1 . Let $E$ be the intersection of the $E_{j}$ and suppose $X$ contains all the $X_{j}$. For sufficiently large $\alpha$ in $E$,

$$
\begin{aligned}
\mu & \left(\left\|\underline{x}_{\alpha} \in \underline{A}\right\| / G(X)\right)=\mu\left(\left\|\underline{x}_{\alpha} \in \bigcup\left\{\underline{A}_{j}: j \in \gamma\right\}\right\| / G(X)\right) \\
& =\mu\left(\sum\left\{\left\|\underline{x}_{\alpha} \in \underline{A}_{J}\right\|: j \in \gamma\right\} / G(X)\right) \\
& =\mu\left(\sum\left\{\left\|\underline{x}_{\alpha} \in \underline{A}_{J}\right\| / G(X): j \in \gamma\right\}\right) \\
& =\sum\left\{\mu\left(\left\|\underline{x}_{\alpha} \in \underline{A}_{j}\right\| / G(X)\right): j \in \gamma\right\}=\sum\left\{r_{J}: j \in \gamma\right\} \\
& =\sum\left\{\nu\left(A_{j}\right): j \in \gamma\right\} .
\end{aligned}
$$

Therefore,

$$
\nu(A)=\lim _{X} \nu_{X}(\underline{A})=\sum\left\{\nu\left(A_{j}\right): j \in \gamma\right\}
$$

where $A$ is the union of the $A_{j}$.

Claim 4. $\nu$ extends the usual product measure.

It suffices to show that $\nu$ agrees with the usual product measure on finite joins of the canonical generators. This is straightforward by the definition of $\mu$ on the $\mathscr{B} / G(X)$ (Fact 3 of $\S 2$ ).

TheOREM. Assume GCH and that $\theta$ is an infinite cardinal of uncountable cofinality.

(1) If $\theta$ is singular then $V^{\mathscr{B}(\theta)} \vDash$ "if $\rho$ is a regular cardinal below $\theta$ and $\mathscr{C}$ is a collection of subsets of ${ }^{\theta} 2$ with fewer than $\theta$ elements then there is a $\rho$-additive extension of the usual product measure on ${ }^{\theta} 2$ to a $\rho$-algebra containing $\mathscr{C} "$.

(2) If $\theta=\lambda^{+}$and $\rho=\operatorname{cof}(\lambda)$ then $V^{\mathscr{B}(\theta)} \vDash$ "if $\mathscr{C}$ is a collection of subsets of ${ }^{\theta} 2$ of size $\lambda$ there is a $\rho$-additive extension of the usual product measure on ${ }^{\theta} 2$ to a $\rho$-algebra containing $\mathscr{C} "$.

(3) If $\theta$ is regular and $\lambda$ is an infinite cardinal with $\lambda^{+}<\theta$ then $V^{\mathscr{B}(\theta)} \vDash$ "if $\mathscr{C}$ is a collection of subsets of ${ }^{\theta} 2$ of size $\lambda$ there is a $\theta$-additive extension of the usual product measure on ${ }^{\theta} 2$ to a $\theta$-algebra containing $\mathscr{C} "$.

Proof. (1) follows immediately from the previous lemma as does (2) provided $\omega<\rho$. (2) with $\omega=\rho$ follows from a well-known result of Horn and Tarski concerning finitely additive measures on Boolean algebras. 
(3) is proved by an argument similar yet simpler than that used to prove the previous lemma. I will indicate the modifications leaving details to the reader. Work with the CBA $\mathscr{B}(\theta \times \theta)$ rather than $\mathscr{B}(\theta)$; in other words, replace the choice of $\kappa=\lambda^{+}$in the proof of the lemma by $\kappa=\theta$. Suppose $G \subseteq \mathscr{B}$ is $V$-generic and in $V[G] \mathscr{C}$ is a collection of subsets of ${ }^{\theta} 2$ of size $\lambda$ where $\lambda^{+}<\theta$. Redefine $S$ to be $\left\{\alpha \in \theta: \operatorname{cof}(\alpha)=\lambda^{+}\right\}$. Instead of a collection of regressive functions there will be a single regressive function $f$ which codes up the behavior of all the elements of $\mathscr{C}$ (more precisely, the behavior of names chosen for the elements of $\mathscr{C}$ ). $\rho$ is replaced everywhere by $\theta$. In particular, $\mathscr{A}$ is now the $\theta$-algebra generated by $\mathscr{C} . \nu_{X}$ is defined as before except that $X$ (and $Y$ ) are now allowed to vary over subsets of $\theta \times \theta$ of size less than $\theta$. The use of the lemma from $\S 3$ is replaced by an application of Fodor's Lemma to the function $f$ which results in a stationary set $E$ which will work uniformly for all $A$ and $\underline{A}$ in claim 1 . The rest of the proof is essentially the same.

Notice that if for any collection $\mathscr{A}$ of subsets of ${ }^{\theta} 2$ of size $\lambda$ there's a $\rho$-additive measure extending the usual product measure which is defined on a $\rho$-algebra containing $\mathscr{A}$ then the same statement is true replacing $\theta$ by any smaller cardinal.

5. The normal Moore space conjecture. The Normal Moore Space Conjecture is that all Normal Moore Spaces are metrizable. The independence of NMSC was discovered by Silver in 1967 using a result of Bing. The consistency of NMSC was proved only recently by Nyikos [6] using a theorem of Kunen. Nyikos' proof used the consistency of strongly compact cardinals and the question remained whether one might obtain the consistency of NMSC from the consistency of ZFC alone. This section shows that a fragment of NMSC can be obtained without the use of large cardinals. After the results of this paper were obtained, Fleissner showed that if there are nonmetrizable normal Moore spaces then there's an inner model with a measurable cardinal. See Tall [10] and Fleissner [2] for a more detailed account of the history of the problem along with proofs.

Nyikos' contribution to settling the NMSC is largely contained in the following theorem.

THEOREM (Nyikos [6]). If the usual product measure on ${ }^{\lambda} 2$ can be extended to a countably additive measure defined on all members of $\mathscr{A}$ for any collection $\mathscr{A}$ of subsets of ${ }^{\lambda} 2$ of size $\lambda$ then every normal Moore space of size $\lambda$ is metrizable. 
Nyikos' theorem and the theorem of the previous section give

TheOREM. Assume GCH. If $\theta$ is a cardinal of uncountable cofinality then $V^{\mathscr{B}(\theta)} \vDash " \theta=2^{\omega}$ and every normal Moore space of size $<2^{\omega}$ is metrizable".

Proof. This follows immediately from Nyikos' theorem and the theorem of $\S 4$ if $\theta$ isn't the successor of a cardinal of cofinality $\omega$.

So suppose $\theta=\lambda^{+}$where $\lambda$ is a cardinal and $\operatorname{cof}(\lambda)=\omega$. As above $V^{\mathscr{B}(\theta)} \vDash$ "every normal Moore space of size $<\lambda$ is metrizable". The problem is with spaces of size $\lambda$. Assume $V^{\mathscr{R}(\theta)} \vDash$ " $\underline{X}$ is a normal Moore space of size $\lambda$ ". We will use the absoluteness arguments between $V^{\mathscr{B}(\theta)}$ and $V^{\mathscr{B}\left(\theta^{+}\right)}$.

Claim $1 . V^{\mathscr{B}\left(\theta^{\dagger}\right)} \vDash$ “ $\underline{X}$ is a normal Moore space”. (In $V^{\mathscr{R}\left(\theta^{\dagger}\right)}, \underline{X}$ is given the topology generated by its open sets in $V^{\mathscr{B}(\theta)}$.)

That $V^{\mathscr{B}\left(\theta^{+}\right)} \vDash$ " $\underline{X}$ is a Moore space" is trivial. We may suppose that $\underline{X}$ has universe $\lambda$. Choose $\underline{D} \in V^{\mathscr{B}(\theta)}$ such that $V^{\mathscr{B}(\lambda)} \vDash$ " $\underline{D}$ is a basis for $\underline{X}$ of size $\lambda$ ". We may assume that $\mathfrak{D}$ is actually in $V^{\mathscr{B}(\lambda)}$ since it consists of $\lambda$ sets each of which has size at most $\lambda$. If $V^{\mathscr{B}\left(\theta^{+}\right)} \vDash$ “ $C \underline{C}$ and $\underline{D}$ are disjoint closed sets of $\underline{X}$ which can't be separated", then for some $\alpha \in\left[\theta, \theta^{+}\right)$, $\underline{C}, \underline{D} \in V^{\mathscr{B}(\alpha)}$. By downward absoluteness $V^{\mathscr{B}(\alpha)} \vDash$ " $\underline{C}$ and $\underline{D}$ are closed sets in the topology generated by $\mathfrak{D}$ which can't be separated". However there's a bijection between $\theta$ and $\alpha$ which is the identity below $\lambda$ giving an isomorphism between $\mathscr{B}(\theta)$ and $\mathscr{B}(\alpha)$ which is the identity on $\mathscr{B}(\lambda)$. This isomorphism extends to an isomorphism from $V^{\mathscr{B}(\theta)}$ to $V^{\mathscr{R}(\alpha)}$ which sends $\stackrel{D}{2}$ to $\mathfrak{D}$. This is a contradiction since $V^{\mathscr{B}(\theta)} \vDash$ "the topology generated by $\mathfrak{D}$ is normal" while $V^{\mathscr{B}(\alpha)} \vDash$ "the topology generated by $\mathfrak{D}$ is not normal". Therefore $V^{\mathscr{B}\left(\theta^{\dagger}\right)} \vDash$ “ $\underline{X}$ is normal".

Claim 2. $V^{\mathscr{P}(\theta)} \vDash$ “ $\underline{X}$ is metrizable".

$V^{\mathscr{B}\left(\theta^{+}\right)} \vDash " \underline{X}$ is metrizable" by the easy case of this proof. By an argument similar to that in Claim 1, the metric on $\underline{X}$ can be pulled down to $V^{\mathscr{B}(\theta)}$.

6. Measure extension axioms and small large cardinals. The proofs of this section use standard techniques or are modifications of proofs of well-known theorems so I will only sketch the arguments.

In some directions the theorem of $\S 4$ is best possible. For example, if $2^{\omega}=\omega_{2}$ then there is a collection $\mathscr{A}$ of subsets of the unit interval of size 
$\omega_{1}$ such that Lebesgue measure has no $\omega_{2}$-additive extension to a $\sigma$-algebra containing $\mathscr{A}$ while the corollary of $\S 3$ shows it is consistent there will always be such a measure which is countably additive instead of $\omega_{2}$-additive. To verify the first part of the previous statement, consider an $\left(\omega_{2}, \omega_{1}\right)$-Ulam matrix (see [4]). Since $2^{\omega}=\omega_{2}$ each row (of size $\left.\omega_{2}\right)$ can be countably generated by a countable collection of sets. So the entire matrix can be countable generated by $\omega_{1}$ sets. By the usual arguments, this provides a collection of sets of reals with the desired properties.

One of the simplest ways one might hope to improve the result of section 3 would be to show the consistency of "Lebesgue measure can be extended to a countably additive measure on any $2^{\omega}$ new sets" without using large cardinals. The next theorem and corollary show this is impossible.

For convenience let $P(\kappa)$ abbreviate the statement "if $\mathscr{A}$ is a collection of subsets of $\kappa$ of size $\kappa$ there is a countably additive measure which is defined on each element of $\mathscr{A}$ and which vanishes on points."

THEOREM 1. If $\kappa$ is the least infinite cardinal such that $P(\kappa)$ holds then $\kappa$ is weakly inaccessible and $\kappa$ has the tree property (i.e., there is no $\kappa$-Aronszajn tree).

Proof. Suppose $\kappa$ is singular. I will describe a collection of $\kappa$ sets which will contradict the definition of $\kappa$. First partition $\kappa$ into $\operatorname{cof}(\kappa)$ many sets each of size less than $\kappa$ and by the definition of $\kappa$ choose a collection of subsets of $\kappa$ which guarantee that some element of the partition must be given positive measure. Now for each element of the partition add a collection of fewer than $\kappa$ sets which guarantee it must have measure zero if all its elements are assigned zero mass.

Suppose $\kappa=\lambda^{+}$. Consider a $\left(\lambda^{+}, \lambda\right)$-Ulam matrix. Choose sets which guarantee no bounded initial segment of $\kappa$ can have positive measure unless some point does. For each of the columns of the matrix choose a family of subsets of the union of the column which guarantees that if the column has positive measure one of its elements must. The usual argument shows this contradicts the definition of $\kappa$.

Assume $T$ is a $\kappa$-Aronszajn tree with universe $\kappa$. Pick a collection of sets which guarantee that no bounded subset of $T$ may have positive measure unless some point has positive mass. Since each level has fewer than $\kappa$ elements there's a collection of sets that guarantees, along with the sets already chosen, that each level contains an element whose successors form a set of positive measure. Let $\nu$ be a countably additive measure on this collection of sets. For some $\varepsilon>0$ the set of nodes whose successors 
form a set of measure at least $\varepsilon$ has size $\kappa$. The tree consisting of these nodes has no infinite antichain so it must have a branch of length $\kappa$.

$\kappa$ can in fact be shown to be Mahlo and beyond using matrices designed by Hajnal. Devlin had noticed that $\kappa$ has the tree property if for every collection $\mathscr{A}$ of subsets of $\kappa$ of size $\kappa$ there is a $\kappa$-additive measure which is defined on all of $\mathscr{A}$ and vanishes on points.

Silver showed that if a regular cardinal has the tree property then it is weakly compact in $L$. Prikry and I noticed that if $\kappa$ is weakly compact then by adding $\kappa$ random reals a model is obtained where Lebesgue measure can be extended to any $2^{\omega}$ sets.

COROLlaRY. ZFC + "Lebesgue measure can be extended to a countably additive measure on any collection of $2^{\omega}$ sets" is equiconsistent with the existence of a weakly compact cardinal.

Theorem 3 of this section is a modification of the argument used to prove the following theorem. A proof is given in [5], page 220. Also see Prikry [8].

THEOREM 2 (Prikry). If $\lambda<\kappa$ are regular cardinals and there is a uniform $\lambda$-indecomposible ultrafilter over $\kappa$ then every stationary set consisting of points of cofinality $\lambda$ reflects, i.e. has an initial segment which is stationary in its sup.

THEOREM 3. If $\kappa$ is minimal such that $P(\kappa)$ holds then every stationary subset of $\kappa$ consisting of points of cofinality $\omega$ reflects.

Proof. Assume $A \subseteq \kappa$ has no stationary initial segments and consists of points of cofinality $\omega$. For each $\alpha \in A$ fix a set $F_{\alpha}$ of order type $\omega$ which is cofinal in $\alpha$. A function $f$ with domain $A \cap \lambda$ is called a disjointer for $A \cap \lambda$ if $[f(\alpha), \alpha) \cap F_{\alpha}$ has empty intersection with $[f(\beta), \beta) \cap F_{\beta}$ whenever $\alpha, \beta \in A \cap \lambda$ are distinct. As in [4], for every $\lambda<\kappa$ there's a disjointer $f_{\lambda}$ for $A \cap \lambda$. To show $A$ is nonstationary it suffices to construct a disjointer for $A$.

Choose a collection of subsets of $\kappa$ which guarantees that no bounded part of $\kappa$ can have positive measure and that for each $\alpha \in A$ there's $\xi \in F_{\alpha}$ such that $\left\{\lambda: f_{\lambda}(\alpha) \leq \xi\right)$ has measure $>1 / 2$. Choose a countably additive measure $\nu$ on this family and define a disjointer $f$ for $A$ by $f(\alpha)=$ the least $\xi \in F_{\alpha}$ such that $\left\{\lambda: f_{\lambda}(\alpha) \leq \xi\right)$ has measure $>1 / 2$. 


\section{REFERENCES}

[1] S. Banach and C. Kuratowski, Sur une generalization du problème de la measure, Fund. Math., 14 (1929), 127-131.

[2] William G. Fleissner, The normal Moore space conjecture and large cardinals, to appear.

[3] Paul R. Halmos, Measure Theory, van Nostrand-Reinholt, Princeton, New Jersey, 1950.

[4] Thomas Jech, Set Theory, Academic Press, New York, San Francisco, London, 1978.

[5] Aki Kanamori and Menachem Magidor, The Evolution of Large Cardinals in Set Theory, in: Higher Set Theory, Lecture Notes in Math. 699, Springer, Berlin, pp. 99-275, 1978.

[6] P. J. Nyikos, A provisional solution to the normal Moore space problem, Proc. Amer. Math. Soc., 78 (1978), 429-435.

[7] Karel Prikry, Measure extension axioms, handwritten notes.

[8] - On Descendingly Incomplete Ultrafilters, in: Cambridge Summer School in Mathematical Logic, Lecture Notes in Math. 377, Springer, Berlin, pp. 459-488, 1973.

[9] R. M. Solovay, Real-valued measurable cardinals, Proceedings Symposia in Pure Mathematics, 13 (1971), 397-428.

[10] Franklin D. Tall, The normal Moore space problem, Topological Structures II, Mathematical Centre Tracts 116, Amsterdam, pp. 243-261, 1979.

[11] _ 'Locally compact perfectly normal spaces are collectionwise normal' is consistent, to appear.

Received December 21, 1982 and in revised form July 14, 1983.

ThE OHIo STATE UNIVERSITY

Columbus, OH 43210 



\title{
PACIFIC JOURNAL OF MATHEMATICS \\ EDITORS
}

\author{
DoNALD BABBITT (Managing Editor) \\ University of California \\ Los Angeles, CA 90024 \\ J. DugunduI \\ University of Southern California \\ Los Angeles, CA 90089-1113 \\ R. FINN \\ Stanford University \\ Stanford, CA 94305 \\ HERMANN FlasChKa \\ University of Arizona \\ Tucson, AZ 85721
}

C. C. Moore

University of California

Berkeley, CA 94720

ARTHUR OGUS

University of California

Berkeley, CA 94720

Hugo Rossi

University of Utah

Salt Lake City, UT 84112

H. SAMELSON

Stanford University

Stanford, CA 94305

ASSOCIATE EDITORS
R. ARENS
E. F. BECKENBACH
B. H. NeUMANN
F. WOLF
K. YoSHIDA (1906-1982)

\section{SUPPORTING INSTITUTIONS}

UNIVERSITY OF ARIZONA
UNIVERSITY OF BRITISH COLUMBIA
CALIFORNIA INSTITUTE OF TECHNOLOGY
UNIVERSITY OF CALIFORNIA
MONTANA STATE UNIVERSITY
UNIVERSITY OF NEVADA, RENO
NEW MEXICO STATE UNIVERSITY
OREGON STATE UNIVERSITY
UNIVERSITY OF ARIZONA
CALIFORNIA INSTITUTE OF TECHNOLOGY
UNIVERSITY OF CALIFORNIA
UNIVERSITY OF NEVADA, RENO
OREGON STATE UNIVERSITY
UNIVERSITY OF OREGON
UNIVERSITY OF SOUTHERN CALIFORNIA
STANFORD UNIVERSITY
UNIVERSITY OF HAWAII
UNIVERSITY OF TOKYO
UNIVERSITY OF UTAH
WASHINGTON STATE UNIVERSITY
UNIVERSITY OF WASHINGTON 


\section{Pacific Journal of Mathematics}

Vol. 115, No. $1 \quad$ September, 1984

Carlos Andradas Heranz and José Manuel Gamboa Mutuberría, A note on projections of real algebraic varieties $\ldots \ldots \ldots \ldots \ldots \ldots \ldots \ldots \ldots \ldots$

Jürgen Appell and Maria Patrizia Pera, Noncompactness principles in nonlinear operator approximation theory $\ldots \ldots \ldots \ldots \ldots \ldots \ldots \ldots \ldots \ldots$

Timothy John Carlson, Extending Lebesgue measure by infinitely many

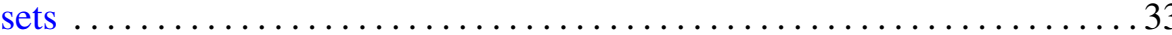

Donald S. Coram and Paul Frazier Duvall, Jr., Non-cell-like

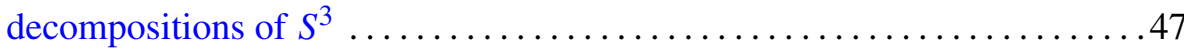

Edward Norman Dancer, Order intervals of selfadjoint linear operators and

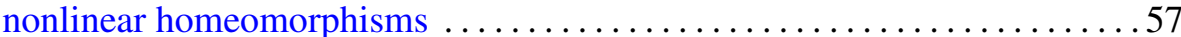

Ralph Jay De Laubenfels, Well-behaved derivations on $C[0,1] \ldots \ldots \ldots 73$

D. Feyel and A. de La Pradelle, Sur certaines extensions du théorème

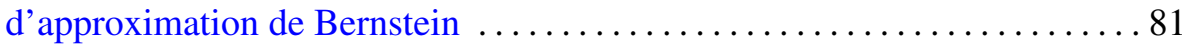

Colin C. Graham and Bertram Manuel Schreiber, Bimeasure algebras on

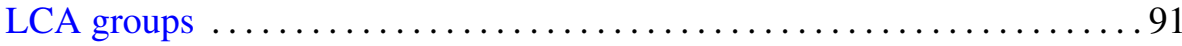

Richard Howard Hudson, Class numbers of imaginary cyclic quartic fields

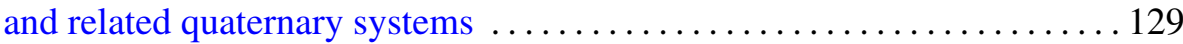

Carl Groos Jockusch, Jr. and Iraj Kalantari, Recursively enumerable sets and van der Waerden's theorem on arithmetic progressions . . . . . . . . 143

J. F. McClendon, On noncontractible valued multifunctions ........... 155 Akihiko Miyachi, Weak factorization of distributions in $H^{p}$ spaces $\ldots \ldots \ldots 165$ Ezzat S. Noussair and Charles Andrew Swanson, Global positive solutions of semilinear elliptic problems

Jon Christopher Snader, Strongly analytic subspaces and strongly

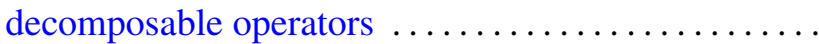

Boguslaw Tomaszewski, A construction of inner maps preserving the Haar measure on spheres

Akihito Uchiyama, The Fefferman-Stein decomposition of smooth functions and its application to $H^{p}\left(\mathbf{R}^{n}\right) \ldots \ldots \ldots$ 